Myokarditis: vielfältige Symptomatik, unterschiedliche Verläufe

\title{
Herz in Flammen
}

\author{
Das klinische Bild der Myokarditis ist sehr heterogen. Das Spektrum \\ reicht von leichter unspezifischer Symptomatik mit guter Prognose bis \\ hin zum fulminanten Verlauf bzw. dem akuten Herztod.
}

_ Unter einer Myokarditis versteht man eine akute oder chronische Inflammationsreaktion des Myokards unterschiedlicher Genese, wobei in unterschiedlichem Maß Kardiomyozyten, Fibroblasten, koronare Arteriolen, Kapillaren und sogar epikardiale Koronararterien einbezogen sein können. Männer sind 1,5fach häufiger betroffen als Frauen. „Die Klinik ist vielgestaltig“, erläuterte PD Guido Michels von der Kardiologischen Universitätsklinik in Köln. Die häufigsten Symptome sind Dyspnoe (40-70\%), Brustschmerzen (30-60\%) und Palpitationen (10-30\%). „Nicht selten imponiert die Erkrankung wie ein akutes Koronarsyndrom“, so Michels. In ca. 5\% entwickelt sich ein kardiogener Schock, und für 20\% der Fälle eines akuten Herztodes bei jungen Erwachsenen ist eine Myokarditis verantwortlich. Die intrahospitale Mortalität einer akuten fulminanten Myokarditis liegt bei 30-50\%. Die 5-Jahres-Mortalität nach Myokarditis beträgt ca. $20 \%$.

\section{Unterschiedlicher Verlauf}

„Bei der Manifestation der Myokarditis spielen neben dem Geschlecht auch Umweltfaktoren, Vitaminmangel, die Jahreszeit und genetische Faktoren eine Rolle“, so Michels. Der Verlauf der Er-

\section{Komplexe Pathogenese}

Die Pathogenese der Myokarditis ist sehr komplex. So kommt es einerseits zu einer direkten viralen Zellschädigung, andererseits aber auch zu einer Virus-vermittelten Autoimmunreaktion. Kann das Virus eliminiert werden, so heilt die Myokarditis aus, persistiert das Virus oder die Autoimmunreaktion so entwickelt sich eine chronische Myokarditis mit oder ohne Ausbildung einer dilatativen Kardiomyopathie. krankung ist individuell sehr unterschiedlich: Bei 50-70\% der Patienten kommt es zur Spontanheilung ohne Residuen, bei $20-30 \%$ entwickelt sich eine dilatative Kardiomyopathie und 10\% der Patienten zeigen einen progressiven Verlauf. Ungünstige Prognosefaktoren sind eine NYHA-Stadium III-IV, eine Einschränkung der $\mathrm{EF}<50 \%$, eine positive Immunhistologie in der Biopsie, ein LGE (Late Gadolinium Enhancement) im MRT und die Nicht-Gabe eines Betablockers. Reizleitungsstörungen im EKG, ein Anstieg von CK und CRP und eine Abnahme der EF müssen auch als ungünstige Zeichen im Hinblick auf die Entwicklung einer fulminanten Myokarditis gewertet werden.

\section{MRT: „All-in-one-Bildgebung"}

„Im Unterschied zum Echo bietet das MRT neben der Beurteilung der Morphologie und Funktionalität des linken Ventrikels auch die Möglichkeit der Gewebecharakterisierung, also eine All-inone-Bildgebung", so Michels. So kann ein Ödem, mittels Early-GadoliniumEnhancement eine Hyperämie und mittels Late-Gadolinium-Enhancement eine Nekrose bzw. Fibrose nachgewiesen werden. Gerade die akute Myokarditis lässt sich im Vergleich zur chronischen Verlaufsform mittels MRT besser diagnostizieren. Ein Normalbefund schließt eine Myokarditis allerdings nicht aus.

Insgesamt basiert die Diagnose „Myokarditis“ auf vier Kriterien: EKG-Veränderungen (Repolarisationsstörungen, Blockierungen oder Arrhythmien), erhöhtes Troponin, regionale Wandbewegungsstörungen bzw. globale systolische oder diastolische Dysfunktion im Echo oder MRT und Gewebeveränderungen im MRT. „Der Verdacht auf eine Myokarditis muss geäußert werden, wenn ein

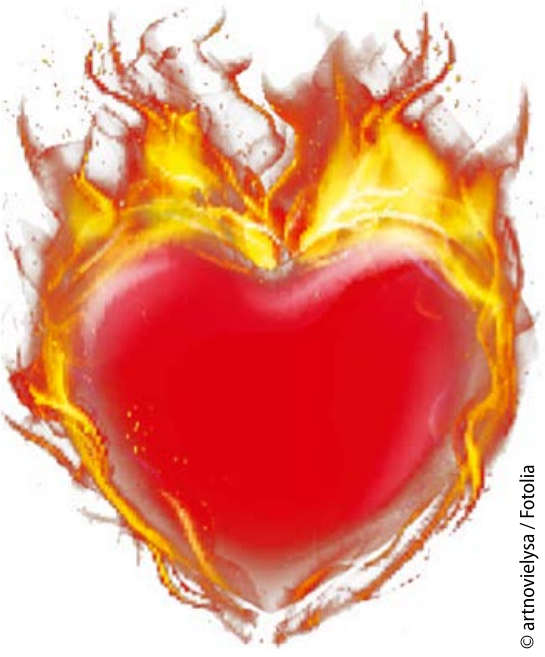

Die Mortalität der fulminanten Myokarditis liegt bei $30-50 \%$.

symptomatischer Patient mindestens eines dieser Kriterien,und ein asymptomatischer Patient mindestens zwei dieser Kriterien aufweist und andere Ursachen wie KHK, Vitien oder Lungenembolie ausgeschlossen sind“, so Michels.

\section{Spezifische Differenzialtherapie}

Zusätzlich sollte eine Endomyokardbiopsie erfolgen. Doch dabei müssen einige Limitationen bedacht werden: Bei fokalem Befall besteht die Möglichkeit eines „sampling error“, die Invasivität geht mit einem Komplikationsrisiko von bis zu 1\% einher, und bei der histologischen Befundung gibt es eine Interobserver-Variabilität. Um eine maximale diagnostische Ausbeute zu erreichen, sollten neben den histologischen Routinefärbungen auch immunhistologische Spezialfärbungen zur Identifizierung von Entzündungszellen und molekularpathologische PCRUntersuchungen zum Nachweis von $\mathrm{Vi}$ rusgenom durchgeführt werden, da sich daraus differenzialtherapeutische Ansätze ergeben. So gibt es bei einer viruspositiven Myokarditis die Option einer Immunmodulation mit einer antiviralen (Herpes-Virus) bzw. Interferon-Therapie (Entero- und Adenoviren). Bei einer virusnegativen Myokarditis empfiehlt sich dagegen eine immunsuppressive Therapie mit Steroiden und Azathioprin.

\section{Dr. Peter Stiefelhagen}

- Quelle: Fortbildungsveranstaltung der Kardiologischen Universitätsklinik in Köln, 9.1.2016 